Mathematical Research Letters 6, 31-41 (1999)

\title{
BEYOND LIOUVILLIAN TRANSCENDENCE
}

\author{
C. Camacho and B. Azevedo Scárdua
}

\begin{abstract}
To a codimension one foliation $\mathcal{F}$ defined by a meromorphic 1 -form $\omega$, one may associate a Godbillon-Vey sequence $\left(\omega_{j}\right), j=0,1, \ldots$, of meromorphic 1 -forms $\omega_{j}$ with $\omega_{0}=\omega$. The sequence is said to have finite length $k$ if $\omega_{k} \neq 0$ and $\omega_{j}=0$ for $j>k$. The case $k=0,1$ or 2 corresponds, respectively, to the case where the foliation $\mathcal{F}$ has additive, affine or projective transverse structure and $k \leq 1$ is equivalent to the existence of a Liouvillian first integral. These are the only possible cases where the transverse structures come from an action of a Lie group on $\overline{\mathbb{C}}$ and a non-trivial model for these foliations is the Riccati differential equation. We propose to go beyond the Lie group transverse structure by studying the case of general $k$ and, for this case, we determine a model differential equation, which generalizes the Riccati equation. We also discuss some other related topics.
\end{abstract}

\section{Introduction}

Let $M$ be a complex manifold and $X \subset M$ an analytic subset of codimension $\geq 2$. A complex codimension one analytic foliation $\mathcal{F}$ on $M$, with singular set $X=\operatorname{sing}(\mathcal{F})$, is defined by a maximal atlas of submersions $y_{i}: U_{i} \rightarrow \mathbb{C}$, covering $M \backslash X$, defining $\mathcal{F}$ outside $X$. This atlas induces a transverse structure cocycle $\left(g_{i j}\right)$, where the $g_{i j}$ are local biholomorphisms of $\mathbb{C}$ defined by $y_{i}=g_{i j}\left(y_{j}\right)$, provided that $U_{i} \cap U_{j} \neq \phi$. In a natural way this cocycle can be regarded as the holonomy pseudogroup of $\mathcal{F}$ defined by the given atlas. The simplest pseudogroup structure of $\mathcal{F}$ is obtained as restriction of a Lie group action on $\overline{\mathbb{C}}$. In this case we have only three possibilities, additive, affine and projective transverse structures (see [5],[17]). A nontrivial model for these foliations is the Riccati differential equation $\mathcal{R}: p(x) d y-\left(a_{2}(x) y^{2}+a_{1}(x) y+a_{o}(x)\right) d x=0$, where the $a_{j}(x)$ and $p(x)$ are polynomials [17]. Indeed, if we take for instance $M$ a simply-connected projective manifold such that the complementary of any analytic codimension one subset is a Stein manifold, then any foliation $\mathcal{F}$ admitting a compact invariant divisor $\Lambda \subset M$ with solvable holonomy, will have a projective structure in a Zariski's open subset of $M$ (containing $\Lambda \backslash \operatorname{sing}(\mathcal{F})$ ) and will be, under generic hypothesis on $\operatorname{sing}(\mathcal{F})$, either a Darboux (linear logarithmic)

Received January 15, 1998.

B. Scárdua supported by CNPq-Brasil.

AMS classification: 32L30,58F18.

keywords: holomorphic foliation, Godbillon-Vey sequence, Lie transverse structure. 
foliation, or the pull back, by a rational map, of a Bernoulli differential equation $([2],[17])$. The linear case corresponds to the case we have a compact invariant divisor with abelian holonomy, [1]. On the other hand it can be proved [2],[17] that foliations with an affine transverse structure outside some compact invariant divisor $\Lambda \subset M$, and satisfying certain (generic) hypothesis on $\operatorname{sing}(\mathcal{F}) \cap \Lambda$, correspond to the ones which have a Liouvillian first integral. More precisely it is shown in [2],[17] that a foliation on $M$, as above, admitting a Liouvillian first integral is in fact either a Darboux foliation or it is the rational pull-back of a suitable Riccati (Bernoulli) differential equation.

The Riccati differential equation was frequently encountered in the classical study of complex differential equations since last century. This is the case, for instance, of the theorem of Painlevé, [14],[15], asserting that a foliation on $\overline{\mathbb{C}} \times \overline{\mathbb{C}}$ with a $n$ to $n$ holonomy map is a pull-back of a Riccati foliation, and also in several other works of P. Painlevé [15]. More recently, a well-known theorem of Malmquist, [10], states that if a complex differential equation on $\overline{\mathbb{C}} \times \overline{\mathbb{C}}$ has a solution which is the graph of a nonalgebraic meromorphic function then $\mathcal{F}$ is a Riccati foliation. This ilustrates the fact that the highest level of transcendence of the solutions of a differential equation with a Lie group transverse structure is contained in the Riccati equation. The purpose of this paper is to go beyond the Lie group transverse structure of a foliation and to enter the unknown domain of the pseudogroup structures. We do that by introducing on this subject the concept of Godbillon-Vey sequences. Assuming that $\mathcal{F}$ is defined by a meromorphic integrable 1 -form $\omega_{o}$, we define a sequence of meromorphic 1-forms $\omega_{0}, \omega_{1}, \ldots$, satisfying certain relations, $d \omega_{0}=\omega_{0} \wedge \omega_{1}, d \omega_{1}=\omega_{0} \wedge \omega_{2}, d \omega_{2}=\omega_{0} \wedge \omega_{3}+\omega_{1} \wedge \omega_{2}, \ldots$ The sequences of "length" 0,1 and 2 , correspond to the transversely additive, affine and projective cases respectively. The general case of finite length $k$ is a sequence $\left(\omega_{j}\right)$, with

$\omega_{k} \not \equiv 0$, and $\omega_{j} \equiv 0, \forall j>k$. The main result of this paper associates to each $k \in \mathbb{N}$ a differential equation which models any foliation with finite GodbillonVey sequence of length $k$ (section 2 ). This differential equation reduces to a Riccati equation whenever $k=0,1,2$. The proposal of this paper is thus to use these models in order to exhibit higher level of transcendence beyond the Liouvillian functions.

Part of this work was done while the authors were visiting the University of Valladolid, we wish to thank J.M. Aroca, F. Cano and M. Carnicer for their warm hospitality. We also wish to acknowledge interesting conversations with R. Moussu and J-F. Mattei.

\section{Transverse structures of holomorphic foliations}

The maximal collection of the $\left(g_{i j}\right)$ defined in the introduction satisfies the cocycle property $g_{i j} \circ g_{j k}=g_{i k}$ and defines the transverse structure of $\mathcal{F}$. The 
foliation $\mathcal{F}$ is called transversely homogeneous if there exists a Lie group $G$ acting on $\overline{\mathbb{C}}$ such that there exists a collection in the transverse structure of $\mathcal{F}$, which consists of restrictions of the biholomorphisms induced by $G$ [6]. For instance we can have $G=(\mathbb{C},+), \operatorname{Aff}(\mathbb{C})$, and $\operatorname{SL}(2, \mathbb{C})$, defining a transversely additive, affine and projective foliation respectively. Indeed these are the only possibilities for a holomorphic codimension-one transversely homogeneous foliation, [5], [17]. Let us fix a meromorphic integrable 1 -form $\omega_{o}$ defining $\mathcal{F}$. In the affine case there exists a global holomorphic 1-form $\omega_{1}$ on $M$ which satisfies $d \omega_{0}=\omega_{0} \wedge \omega_{1}$ and $d \omega_{1}=0$. In the projective case if there exists $\omega_{1}$ such that $d \omega_{0}=\omega_{0} \wedge \omega_{1}$, then there exists also a meromorphic 1-form $\omega_{2}$ in $M$ such that we have $d \omega_{1}=\omega_{0} \wedge \omega_{2}$ and $d \omega_{2}=\omega_{1} \wedge \omega_{2},[6],[1]$. These equations verified in the three cases above are particular cases of a set of relations which we proceed to introduce now.

Let $X$ be a (meromorphic) vector field on $M$ satisfying $\omega_{0}(X)=1$. Recall that the Lie derivative is given by $L_{X}\left(\omega_{0}\right)=i_{X} d \omega_{0}+d\left(i_{X} \omega_{0}\right)$. We consider $\omega_{j}=L_{X}^{j}\left(\omega_{o}\right)$, where $L_{X}^{j+1}(\cdot)=L_{X}\left(L_{X}^{j}(\cdot)\right)$. This construction is found in [7] for the nonsingular real case. The sequence $\omega_{0}, \omega_{1}, \ldots$, of meromorphic 1 -forms thus obtained satisfies the relations:

$$
(E)_{j}: d \omega_{j}=\omega_{0} \wedge \omega_{j+1}+\sum_{k=1}^{j}\left(\begin{array}{l}
j \\
k
\end{array}\right) \omega_{k} \wedge \omega_{j+1-k} .
$$

Any such a sequence is called a Godbillon-Vey sequence.

Denote by $\widehat{\mathcal{X}}(\mathbb{C}, 0)$ the Lie algebra of the formal vector fields in one complex variable with a singularity at the origin, endowed with the Lie bracket. According to [7] the only finite Godbillon-Vey sequences $\left\{\omega_{o}, \ldots, \omega_{k}, 0, \ldots\right\}$, defining Lie algebras, occur in the cases $k=0,1,2$. Moreover the full Godbillon-Vey relations define the Lie algebra $\widehat{\mathcal{X}}(\mathbb{C}, 0)$. In particular there are no Lie algebras associated to finite Godbillon-Vey sequences, strictly between $S L(2, \mathbb{C})$ and $\mathcal{L}(\widehat{\mathcal{X}}(\mathbb{C}, 0))$.

\section{Models of finite length}

Suppose that $\mathcal{F}$ is defined by a meromorphic integrable 1 -form $\omega_{o}$ on a rational algebraic manifold $M$, and that there exists a meromorphic vector field $X$ such that $\omega_{o}(X)=1$. We say that the sequence $\left(\omega_{j}\right)_{j=1}^{\infty}$, where $\omega_{j}=L_{X}^{j}\left(\omega_{o}\right)$, has finite length $k$ if $\omega_{k} \not \equiv 0$ and $\omega_{j} \equiv 0, \forall j>k$.

Theorem 1. The 1-form $\omega_{k}$ is closed and there are two possibilities:

(i) $\omega_{k}$ admits a meromorphic first integral and then there exists a meromorphic map $\pi: M \rightarrow \overline{\mathbb{C}} \times \overline{\mathbb{C}}$ and a model equation of length $k$ in $\overline{\mathbb{C}} \times \overline{\mathbb{C}}$

$$
\mathcal{S}_{k}: \eta_{k}=d y-\left(a_{o}(x) y^{k}+\cdots+a_{k-1}(x) y+a_{k}(x)\right) d x=0,
$$


where the $a_{j}(x)$ are meromorphic functions, such that $\omega_{o}=\pi^{*}\left(\eta_{k}\right)$.

(ii) $\omega_{k}$ does not admit a meromorphic first integral and then we have

$$
\omega_{0}=d f+\left(f^{k} \lambda_{0}+\cdots+\lambda_{k}\right) \omega_{k}
$$

for some meromorphic function $f$ on $M$ and for some constants $\lambda_{0}, \ldots, \lambda_{k} \in$ $\mathbb{C}, \lambda_{k} \neq 0$.

Proof. First we prove that $\omega_{k}$ is closed.

Choose an open set $U \subset M$, nonvoid, such that $\left.X\right|_{U}$ is holomorphic nonsingular and has a flowbox, that is, there are coordinates $\left(x_{1}, \ldots, x_{n}, y\right) \in U$ such that in these coordinates we have $X=\frac{\partial}{\partial y}$. For simplicity of our notation we assume that $n=1$ and write $x_{1}=x$. The condition $i_{X} \omega_{0}=1$ therefore, implies that $\omega_{0}=d y-R(x, y) d x$, where $R$ is meromorphic in $U$. Thus it is easy to see that we have, $\omega_{j}=L_{X}^{j} \omega_{0}=(-1)^{j+1} \frac{\partial^{j}}{\partial y^{j}} R(x, y) d x$. Thus $\omega_{k}=0$ implies that $R$ is polynomial of degree $k$ in the variable $y$, say,

$$
R(x, y)=\sum_{j=1}^{k} a_{j}(x) y^{j}
$$

and in particular $\omega_{k}=k ! a_{k}(x) d x$ depends only on the variable $x$ and is therefore closed.

Now we prove (i): Let us first assume that $\omega_{k}=d x$ for a meromorphic nonconstant function $x: M \rightarrow \overline{\mathbb{C}}$.

Claim. $\omega_{j} \wedge d x \equiv 0, \forall 1 \leq j \leq k$.

Proof. The proof is by induction on $j$ from $j=k$ to $j=1$. We take the equations

$$
(\mathrm{E})_{j}: d \omega_{j}=\omega_{0} \wedge \omega_{j+1}+\sum_{k=1}^{j}\left(\begin{array}{l}
j \\
k
\end{array}\right) \omega_{k} \wedge \omega_{j+1-k},
$$

for $j=1, \ldots, 2 k-2$. For $j=2 k-2$ it gives $\omega_{k} \wedge \omega_{k-1} \equiv 0$ because $\omega_{\ell} \equiv 0, \forall \ell \geq$ $k+1$. Thus we have that the claim holds for $j=k-1$. Assume now that we have proved that $\omega_{j} \wedge d x \equiv 0, \forall j=k-r+1, \ldots, k$. Then using the equation $(\mathrm{E})_{2 k-1-r}$ we conclude that $\omega_{k-r} \wedge 0$. Thus the claim follows by induction.

The claim above and the equations $(\mathrm{E})_{j}$ give:

$$
d \omega_{j}=\omega_{o} \wedge \omega_{j+1}, \forall j=1, \ldots, k .
$$

Now we proceed the proof of (i). According to [17] we can assume $k \geq 3$. Using the claim above we write $\omega_{k-1}=y d x$ for some meromorphic function $y: M \rightarrow \overline{\mathbb{C}}$. Since $\omega_{k} \not \equiv 0$, it follows that $y$ is not constant on $M$. Moreover 
since $M$ is algebraic rational it follows from the claim that $\omega_{j}=h_{j} d x, \forall j=$ $1, \ldots, k-1$. First we assume that $x$ is primitive, i.e., it has connected fibers. We have $\omega_{k-2}=\omega_{o} \wedge y d x=\omega_{o} \wedge d\left(\frac{y^{2}}{2} d x\right)$. Now we write $\omega_{k-2}=h_{2} d x$ for some meromorphic function $h_{2}$ on $M$. Therefore it follows that $d\left(\left(h_{2}-\frac{y^{2}}{2}\right) d x\right) \wedge \omega_{o} \equiv 0$. This implies that $d\left(\left(h_{2}-\frac{y^{2}}{2}\right) d x\right)=g_{2} \omega_{o}$ for some meromorphic function $g_{2}$, and moreover since the left hand 1-form is closed it follows that $g_{2} \omega_{o}$ is also closed. By the hypothesis it implies that $g_{2} \equiv 0$ and therefore $d\left(h_{2}-\frac{y^{2}}{2}\right) \wedge d x \equiv 0$. Since we have chosen $x$ to be primitive it follows that $h_{2}=\frac{y^{2}}{2}+a_{2}(x)$ for some rational function $a_{2}(z)$ on $\overline{\mathbb{C}}$. Thus we have $\omega_{k-2}=\left(\frac{y^{2}}{2}+a_{2}(x)\right) d x$. Now using the induction and the same arguments above we can prove that

$$
\omega_{k-j}=(-1)^{j} k(k-1) \cdots(j+1) j \frac{y^{j}}{k !}\left(\sum_{\ell=1} a_{\ell}(x) \frac{y^{\ell}}{\ell !}\right) d x, \forall j=1, \ldots, k-1,
$$

where $a_{\ell}(z)$ is a rational function on $\overline{\mathbb{C}}$. In particular it follows that $\omega_{1}=$ $\left(k y^{k-1} \alpha_{o}(x)+(k-1) y^{k-2} \alpha_{1}(x)+\cdots+\alpha_{k}(x)\right) d x$ for some meromorphic functions $\alpha_{j}(z)$ on $\overline{\mathbb{C}}$. The first equation $d \omega_{o}=\omega_{1} \wedge \omega_{o}$ now gives that $\omega_{o}=d y-\left(\alpha_{o}(x) y^{k}+\right.$ $\left.\alpha_{1}(x) y^{k-1}+\cdots+\alpha_{k-1}(x) y+\alpha_{k}(x)\right) d x+b(x) d x$, for some rational function $b(z)$ on $\overline{\mathbb{C}}$.

Now, for the general case we recall that according to the Stein Fatorization Theorem [8] (since the leaves of a foliation are always connected) there exists a primitive rational map $x: M \rightarrow \overline{\mathbb{C}}$, and a rational function $a_{k}(z)$ on $\overline{\mathbb{C}}$, such that $\omega_{k}=(-1)^{k+1} k ! a_{k}(x) d x$. Using now essentialy the same arguments above one can prove this case. This proves the first part of the theorem.

Part (ii) in the theorem is proved the same way that the first part. The difference is that since by the hypothesis there exists no rational first integral for $\omega_{k}$, the functions $a_{j}$ we obtain in (i) are constant.

Remark 1. When $M=\overline{\mathbb{C}} \times \overline{\mathbb{C}}$, there exists an independent way of finding these models, using the approach of P. Painlevé [14]. In his memoire Painlevé used frequently the fact that a foliation on $\overline{\mathbb{C}} \times \overline{\mathbb{C}}$, which is transverse to a generic vertical line $\overline{\mathbb{C}}_{x_{o}}=\left\{x_{o}\right\} \times \overline{\mathbb{C}}$ is in fact a Riccati differential equation. The next case to this is the case of foliations which are transverse to generic vertical lines $\overline{\mathbb{C}}_{x_{o}}$, except at infinity, where there is a tangency with these lines of order $k-2$. These are precisely the models $\mathcal{S}_{k}$.

The following theorem, whose proof is easy from [2],[17], shows that for $k \geq 3$ a dense subset of differential equations in $\mathcal{S}_{k}$ have no Liouvillian first integral.

Theorem 2. The set of differential equations in $\mathcal{S}_{k}$ which are not rational pull-backs of Riccati differential equations, form a second category subset of the space $\mathcal{S}_{k}$ on the space of foliations on $\overline{\mathbb{C}} \times \overline{\mathbb{C}}$. 
Remark 2. Perhaps the simplest example where a model $\mathcal{S}_{k}$ is present is the saddle-node situation:

$$
\eta_{k}=x^{p+1} d y-\left[\left(1+\lambda x^{p}\right) y+a_{2}(x) y^{2}+\cdots+a_{k}(x) y^{k}\right] d x=0, k \geq 3 .
$$

It would be interesting to find the natural mathematical object generalizing a Liouvillian function through this example. A natural question is what are the sectorial isotropies of Martinet-Ramis [11] associated to $\eta_{k}=0$.

\section{Holonomy and Godbillon-Vey sequences}

We consider a germ of holomorphic singular foliation $\mathcal{F}$ on $\left(\mathbb{C}^{2}, 0\right)$ given by a holomorphic 1-form $\omega$, defined in a neighborhood $U$ of the origin. Let $S$ be a local smooth separatrix of $\mathcal{F}$ through 0 and choose a local coordinate system $(x, y) \in U$, such that $S=(y=0)$. In a small disk $\overline{\mathbb{D}} \subset S$ we consider the simple path $\gamma(t)=\left(e^{2 \pi i t}, 0\right), t \in[0,2 \pi]$ so if $\Sigma=\{(1, y)\}$, then $\Sigma \cap S=\{(1,0)\}=\left\{p_{1}\right\}$ and the product $\Pi=\gamma([0,2 \pi i]) \times \Sigma$ is a solid torus which is foliated by a transversely holomorphic real flow $\mathcal{L}$, obtained from $\mathcal{F}$, this flow $\mathcal{L}$ has a closed orbit which corresponds to the curve $t \mapsto \gamma(t)$ and whose Poincaré Map is a holomorphic map $h: \Sigma \rightarrow \Sigma$ which fixes the point $p_{1}$. This map is called holonomy map of the separatrix $S$. The identification $\left(\Sigma, p_{1}\right)=(\mathbb{C}, 0)$ allows us to consider $h$ as an element of $\operatorname{Diff}(\mathbb{C}, 0)$.

Problem. Describe the relation between $h$ and the transverse structure of $\mathcal{F}$, by means of the Godbillon-Vey sequence associated to $\omega$.

We consider the natural holomorphic coordinate induced by $y$ on $\Sigma$ and obtain a convergent series

$$
h(y)=\sum_{j=1}^{+\infty} a_{j} y^{j} \in \mathbb{C}\{\{y\}\},
$$

for $h$ in this coordinate. On the other hand we define $X=\frac{\partial}{\partial y}$ a holomorphic vector field in $U$ and write $\omega=-P d x+Q d y, P, Q \in \mathcal{O}(U)$, so that $\omega_{0}=d y-\frac{P}{Q} d x$ defines the same foliation, and $\omega_{0}(X)=1$. Now proceeding as in the GodbillonVey algorithm we obtain a meromorphic sequence for $\mathcal{F}$ in $U$ which is in fact given by,$\omega_{j}=(-1)^{j+1} \frac{\partial^{j}}{\partial y^{j}}(R) d x$, where $R:=\frac{P}{Q}$ is meromorphic. The map $h$ is therefore described as follows: Let $\phi(t, y)$ be the flow $\mathcal{L}$ on $\Pi$, that is, it is the solution of the real differential equation

$$
\frac{d y(t)}{d t}=R(\gamma(t), y(t)) \gamma^{\prime}(t), \gamma(t)=e^{i t}
$$

satisfying the initial condition $y\left(0, y_{0}\right)=y_{0}$. Then the holonomy map is given by $h(y)=\phi(2 \pi, y)$. We also remark that since $\gamma(t)$ is a solution we have $\phi(t, 0)=0$, 
for all $t$. Given any $y \in \Sigma$ we define the path $\gamma_{y}(t)=\left(e^{i t}, \phi(t, y)\right) 0 ; t \in[0,2 \pi]$. Thus $\gamma=\gamma_{0}$. We have :

$$
\frac{\partial \phi}{\partial t}(t, y)=R\left(e^{i t}, \phi(t, y)\right) i e^{i t}
$$

so that

$$
\frac{\partial}{\partial t}\left(\frac{\partial \phi}{\partial y}(t, y)\right)=\frac{\partial R}{\partial y}\left(\gamma_{y}(t)\right) \frac{\partial \phi}{\partial y}(t, y) i e^{i t}
$$

and then the solution is

$$
\frac{\partial \phi}{\partial y}(t, y)=\exp F_{1}(t, y)
$$

where we define,

$$
F_{1}(t, y)=\int_{\gamma} \omega_{1}
$$

and we also define $f_{1}(x)=\int_{1}^{x} \omega_{1}(x, 0)$.

Thus, we have

$$
h^{\prime}(0)=\exp \int_{\gamma} \omega_{1}
$$

(recall that $\phi(0, y)=y)$.

Derivating (2) we obtain:

$$
\frac{\partial}{\partial t}\left(\frac{\partial^{2} \phi}{\partial y^{2}}\right)=\frac{\partial^{2} R}{\partial y^{2}}\left(\gamma_{y}(t)\right)\left(\frac{\partial \phi}{\partial y}(t, y)\right) i e^{i t}+\frac{\partial R}{\partial y}\left(\gamma_{y}(t)\right) \frac{\partial^{2} \phi}{\partial y}(t, y) e^{i t}
$$

Remark 3. The solution of the differential equation

$$
f^{\prime}=a f+b,
$$

is $f=A \int \frac{b}{A}$ where $A=\exp \int a$.

Thus, we obtain

$$
\frac{\partial^{2} \phi}{\partial y^{2}}(t, y)=-\exp F_{1}(t, y) \int_{\gamma_{y}} F_{2}(t, y)
$$

where

$$
F_{2}(t, y)=\int_{\gamma_{y}} \exp F_{1}(t, y) \omega_{2},
$$


we also define $f_{2}(x)=-\exp f_{1}(x) \int_{1}^{x} \omega_{2}(x, 0)$.

Thus, we obtain,

$$
h^{(2)}(0)=\exp \int_{1}^{x} \omega_{1} \int_{1}^{x} \exp f_{1} \omega_{2}
$$

Proceeding this same way we obtain for the $k$-th step:

$$
\frac{\partial^{k} \phi}{\partial y^{k}}(t, y)=\left[\exp F_{1}(t, y)\right] \cdot F_{k}(t, y)
$$

and $h^{(k)}(0)=\left[\exp \int_{\gamma}\right] \cdot \omega_{1} \int_{\gamma} \Omega_{k}$, for some $F_{k}(t, y), f_{k}(x)$ and some differential 1-form $\Omega_{k}$ which we describe inductively below, but first we need the following well-known lemma:

Lemma 1. Let $f, g$ be $C^{\infty}$ real functions such that the composition $f \circ g$ is defined, then:

$$
\frac{1}{k !}(f \circ g)^{(k)}(x)=\sum_{p=1}^{k} \frac{1}{p !} f^{(p)}(g(x)) \sum_{n \in \mathbb{N}(p, k)}\left(\begin{array}{c}
k-1 \\
p-1
\end{array}\right)\left(\frac{g^{(1)}(x)}{1 !}\right)^{n_{1}} \cdots\left(\frac{g^{(k)}(x)}{k !}\right)^{n_{k}},
$$

where $\mathbb{N}(p, k):=\left\{\left(n_{1}, \ldots, n_{k}\right) ; \sum n_{j}=p, \Sigma j n_{j}=k\right\}$, for all $k \in \mathbb{N}$.

Using the formula above we obtain the following:

$$
\begin{aligned}
& \frac{1}{n !} \cdot \frac{\partial^{n}}{\partial y^{n}}\left(\frac{\partial \phi}{\partial t}(t, y)\right)= \\
& {\left[\sum_{p=1}^{n} \frac{1}{p !} \frac{\partial^{p} R}{\partial y^{p}}\left(e^{(i t)}, \phi(t, y)\right) \sum_{k \in \mathbb{N}(n, p)}\left(\begin{array}{c}
k-1 \\
p-1
\end{array}\right)\left(\frac{1}{1 !} \frac{\partial \phi}{\partial y}(t, y)\right)^{\left(k_{1}\right)} \ldots\right.} \\
& \left.\quad\left(\frac{1}{n !} \frac{\partial^{n} \phi}{\partial y^{n}}(t, y)\right)^{k_{n}}\right] i e^{i t}
\end{aligned}
$$

Now we remark that if $\left(n_{1}, \ldots, n_{k}\right) \in \mathbb{N}(p, k)$ is such that $\sum_{j=1}^{k} j n_{j}=n$, then, if $n_{k} \neq 1$, we must have: $n_{k}=1$ and $n_{j}=0$ for the others. Moreover $\sum_{j=1}^{k} n_{j}=1$ and then using also (11) we obtain :

$$
\begin{aligned}
\frac{\partial^{n} \phi}{\partial y^{n}}(t, y)= & {\left[\exp _{1}(t, y)\right] \cdot \int_{\gamma_{y}} \sum_{p=1}^{n} \frac{\partial^{(p)} R}{\partial y^{(p)}\left(\gamma_{y}(t)\right)} \sum_{n \in \mathbb{N}(p, k)}\left(\begin{array}{c}
n \\
p
\end{array}\right) \frac{(n-1) !}{(p-1) !} \times } \\
& \left(\exp _{1}(t, y)\right)^{p-1}\left(\frac{F_{2}(t, y)}{2 !}\right)^{n_{2}} \cdots\left(\frac{F_{n-1}(t, y)}{(n-1) !}\right)^{n_{k-1}} i e^{i t} d t
\end{aligned}
$$


Using this we get the inductive definition for $F_{n}$ and therefore for $f_{n}(x)$ and $\Omega_{n}$, these expressions can be used to prove that:

Lemma 2. $\Omega_{n}(x)$ is a closed Liouvillian differential 1-form on $\{y=0\}$ for every $n$.

Proof. As we have remarked above, this is a consequence of the recurrence relations stated above, but we can give an intuition of the geometric reason; in fact all one need to recall is that the periods $\int_{\gamma} \Omega$ are defined intrinsically by the equations above so that in particular they do not depend on the chosen closed path $\gamma$ on $(y=0)$ as above. This is enough to assure that the restriction $\left.\Omega_{n}\right|_{(y=0)}$ is closed.

Definition 1. The realization equations for the diffeomorphism $h(y)$ are the equations (RE1),(RE2) and (RE3) below:

$$
\begin{gathered}
h^{(1)}(0)=\exp \int_{\gamma} \omega_{1}(x, 0), \\
h^{(k)}(0)=h^{(1)}(0) \int_{\gamma} \Omega_{k}, \\
f_{k+1}(x)=\int_{\gamma} \Omega_{k}(x), \\
\Omega_{k}=\sum_{p=1}^{n}(-1)^{p+1} \sum_{n \in \mathbb{N}(p, k)}\left(\begin{array}{l}
n \\
p
\end{array}\right) \frac{(n-1) !}{(p-1) !} \times\left(\exp f_{1}(x)\right)^{p-1}\left(\frac{f_{2}(x)}{2 !}\right)^{n_{2}} \ldots \\
\quad\left(\frac{f_{n-1}(x)}{(n-1) !}\right)^{n_{k-1}} \omega_{p}(x, 0) .
\end{gathered}
$$

Remark 4. The realization equations introduced above may be applied in order to give a proof of the local synthese result [16], and the main idea is that the convergence of the series

$$
h(y)=\sum_{j=0}^{+\infty} \frac{h^{(j)}(0)}{j !} y^{j}
$$

may be used to prove the convergence of a suitable (formal) expression $R(x, y)$ solution for the realization equations.

Remark 5 (Finite length groups): We recall that a germ of foliation $\mathcal{F}$ at $0 \in \mathbb{C}^{2}$, singular at the origin, is not-much degenerate (n.m.d. for short) if: (i) $\mathcal{F}$ is nondicritical and can be desingularized with one sole blow-up, $\pi$ : $\left(\widetilde{\mathbb{C}^{2}}, \mathbb{P}\right) \rightarrow\left(\mathbb{C}^{2}, 0\right)$, (ii) the singularities of the foliation $\widetilde{\mathcal{F}}=\pi^{*} \mathcal{F}$, appearing over 
the invariant projective line $\mathbb{P}=\pi^{-1}(0)$ are of the reduced form $x d y-\lambda y d x+$ $\ldots, \lambda \notin \mathbb{Q}_{+}$, for some local chart with $\mathbb{P}=(y=0)$. In this case we consider the holonomy group of the leaf $\mathbb{P} \backslash \operatorname{sing} \widetilde{\mathcal{F}}$; which is called the projective holonomy of $\mathcal{F}$ (see [3]). In [3] we study the analytical invariants of such foliations. We recall that two germs of foliations $\mathcal{F}, \mathcal{F}_{1}$ at $0 \in \mathbb{C}^{2}$ are strongly analytically equivalent if there exists a germ of biholomorphism $\widetilde{\Phi}:\left(\tilde{\mathbb{C}}^{2}, \mathbb{P}\right) \rightarrow\left(\tilde{\mathbb{C}}^{2}, \mathbb{P}\right)$ which is an equivalence for $\tilde{\mathcal{F}}$ and $\tilde{\mathcal{F}}_{1}$. Such an equivalence induces a strong analytical equivalence $\Phi:\left(\mathbb{C}^{2}, 0\right) \rightarrow\left(\mathbb{C}^{2}, 0\right)$ between $\mathcal{F}$ and $\mathcal{F}_{1}[3]$. In [3] we prove that if two germs of n.m.d. foliations have their separatrices contained in complex lines, the same projective singular points and singularities, and conjugated projective holonomy groups, then they are strongly analytically equivalent. We also prove that any finitely generated subgroup of $G \subset \operatorname{Diff}(\mathbb{C}, 0)$ is conjugated to the projective holonomy group of a n.m.d germ $\mathcal{F}(G)$ called a realization of $G$, which has separatrices contained in complex lines. Finally, from [1],[2],[9],[13],[17] we have that if $G \subset \operatorname{Diff}(\mathbb{C}, 0)$ is a finitely generated subgroup and $\mathcal{F}(G)$ is its realization then:

(i) $G$ is finite $\Leftrightarrow \mathcal{F}(G)$ has a local holomorphic first integral $f \in \mathcal{O}_{2}$;

(ii) $G$ is abelian linearizable $\Leftrightarrow \mathcal{F}(G)$ is a germ of Darboux (logarithmic) foliation $\sum_{j} \lambda_{j} \frac{d f_{j}}{f_{j}}=0$

(iii) $G$ is abelian analytically normalizable $\Leftrightarrow \mathcal{F}(G)$ is given by a closed meromorphic 1-form $\omega$;

(iv) $G$ is solvable analytically normalizable $\Leftrightarrow \mathcal{F}(G)$ is given by a closed meromorphic 1-form or a pull-back of a germ of Riccati foliation (i.e., of model $\mathcal{S}_{2}$ ).

Motivated by these facts we introduce the following definition:

Definition 2. A finitely generated subgroup of $\operatorname{Diff}(\mathbb{C}, 0)$ has finite length $k$ if its realization $\mathcal{F}(G)$ is of model $\mathcal{S}_{k}$.

Nakai's Theorem [12] and the above remark imply that if $\mathcal{F}(G)$ has closed leaves outside its set of separatrices, then $\mathcal{F}(G)$ is of model $\mathcal{S}_{k}, k \leq 2$ [4]. Also according to [2],[17] if $\mathcal{F}(G)$ admits a Liouvillian first integral, then it is of model $\mathcal{S}_{k}, k \leq 2$. The question now is to decide whether there exists some dynamical or some integration hypothesis on the foliation $\mathcal{F}(G)$, which assures that a subgroup $G \subset \operatorname{Diff}(\mathbb{C}, 0)$, has finite length $k \geq 3$. This problem remains open.

\section{References}

[1] C. Camacho, A. Lins Neto, and P. Sad, Foliations with algebraic limit sets, Ann. of Math. 136 (1992), 429-446.

[2] C. Camacho, and B. Scárdua, Liouvillian first integrals, solvable holonomy groups and Riccati foliations, preprint, IMPA. 
[3] The transcendence of the solutions of a complex differential equation, Ecuaciones Diferenciales — Singularidades (J. Mozo, ed.), Publicaciones de la Universidad de Valladolid, 1997.

[4] Complex foliations with algebraic limit sets, to appear in Astérisque.

[5] E. Ghys, Feuilletages holomorphes de codimension un sur les espaces homognes complexes, Ann. Fac. Sci. Toulouse Math. (6) 5 (1996), 493-519.

[6] C. Godbillon, Feuilletages: Études Geométriques I, Université Louis Pasteur, Mai, 1985.

[7] C. Godbillon and J. Vey, Un invariant des feuilletages de codimension un, C. R. Acad. Sci. Paris Sr. A-B 273 (1971), 92-95.

[8] H. Grauert, R. Remmert, Theory of Stein spaces, Springer-Verlag, Berlin, 1979.

[9] J.F. Mattei and R. Moussu, Holonomie et intégrales premières, Ann. Sci. École Norm. Sup. (4) 13 (1980), 469-523.

[10] J. Malmquist, Sur les functions à un nombre fini des branches définies par les équations différentielles du premier ordre, Acta Math. 36 (1913), 297-343.

[11] J. Martinet and J-P. Ramis, Problème de modules pour des équations différentielles non lineaires du premier ordre, Inst. Hautes Études Sci. Publ. Math. 55 (1982), 63-124.

[12] I. Nakai, Separatrices for non solvable dynamics on $\mathbb{C}, 0$, Ann. Inst. Fourier (Grenoble) 44 (1994), 569-599.

[13] E. Paul, Formes singuliéres à holonomie résoluble, Prépublication Univ. Paul Sabatier, Toulouse, 1995.

[14] P. Painlevé, Leçons sur la théorie analytique des équations différentielles, Librairie Scientifique A. Hermann, Paris, 1897.

[15] _ Ouvres de Paul Painlevé, Tome II, Éditions du Centre National de la Recherche Scientifique, 15, quai Anatole-France,75700, Paris, 1974.

[16] R.Perez Marco and J.C. Yoccoz, Germes de feuilletages holomorphes à holonomie prescrite, Astérisque 222 (1994), 345-371.

[17] B. Azevedo Scárdua, Transversely affine and transversely projective foliations, Ann. Sci. École Norm. Sup. (4) 30 (1997), 169-204.

[18] Integration of complex differential equations, to appear in Journal of Dynamical and Control Systems.

IMPA - Estrada Dona CAstorina 110 - JARdim BotÂnico, CEP 22460-320 — Rio DE JANEIRO — RJ, BRASIL

E-mail address: camacho@euler.impa.br, scardua@impa.br 